\title{
La double perception du sable en Égypte ancienne
}

The double perception of sand in Ancient Egypt

Julie Misuriello

\section{OpenEdition}

Journals

Édition électronique

URL : https://journals.openedition.org/tc/7212

DOI : $10.4000 /$ tc. 7212

ISSN : 1952-420X

\section{Éditeur}

Éditions de l'EHESS

\section{Édition imprimée}

Date de publication : 15 décembre 2013

Pagination : $42-59$

ISBN : 978-2-7351-1654-6

ISSN : 0248-6016

\section{Référence électronique}

Julie Misuriello, «La double perception du sable en Égypte ancienne », Techniques \& Culture [En ligne], 61 | 2013, mis en ligne le 15 décembre 2016, consulté le 29 septembre 2022. URL : http:// journals.openedition.org/tc/7212 ; DOI : https://doi.org/10.4000/tc.7212 


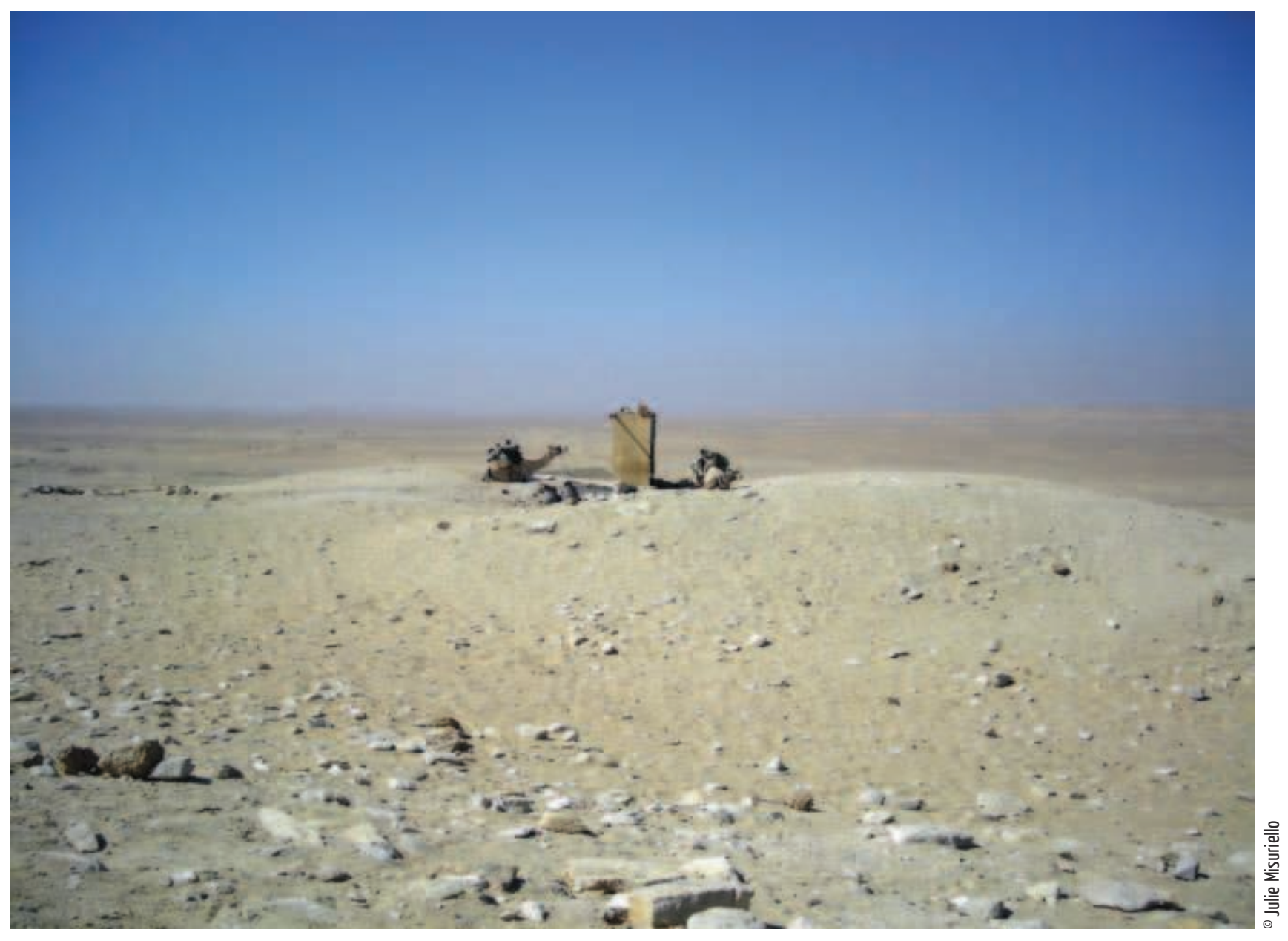




\section{Julie Misuriello}

Laboratoire ASM-ENiM UMR 5140 (CNRS-Montpellier III)

Labex ARCHIMEDE, programme «Investissement d'Avenir »

ANR-11-LABX-0032-01

juliemisuriello@gmail.com
Vivre le sable! Corps, matière et sociétés - 1

Techniques \& Culture 61, 2013/2: $42-59$

\section{LA DOUBLE PERCEPTION DU SABLE EN ÉGYPTE ANCIENNE}

Les Anciens Égyptiens distinguaient Kémet, la «terre noire », fertile, de déchéret, « la terre rouge ", désertique. Un terme unique nomme le sable en égyptien ancien, $\breve{s}(y)$, et le différencie en cela de la terre. À celui-ci viennent s'ajouter les vocables désignant

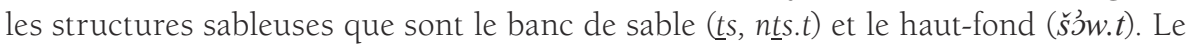
sable, élément principal du milieu hostile qui borde la terre fertile de la Vallée, lieu de vie des Égyptiens, a été étroitement relié à l'idée d'espace désertique. Les sources textuelles évoquent ainsi le «sable du désert » à l'aide du syntagme $\check{s}^{\prime} y n(y)$ h’̉s.t. Dans ce contexte, le sable est également associé à l'étranger par le biais des Bédouins, désignés comme « ceux qui sont sur le sable» $(H r y \cdot w-s ̌ y)$ ou, plus rarement, comme « ceux qui parcourent le sable » (Nmy.w-šs). À côté de ce sable désertique, le lexique égyptien fait également état du « sable de la rive », šy $n(y)$ wd $d b$, sable alluvial, charrié par le fleuve. Mélangé à d'autres éléments, ce sable constituait un matériau fertile recherché. D'emblée, le sable est donc caractérisé par une dualité de nature qui trouve son origine dans la géographie contrastée du territoire égyptien. Il faut toutefois préciser qu'excepté pour certains exemples précis, les textes évoquent généralement l'emploi de «sable », sans préciser sa nature ni son origine.

En s'appuyant sur des recherches menées dans le cadre de la préparation d'une thèse de doctorat portant sur le sable dans les sources sacrées de l'Égypte ancienne ${ }^{1}$, il s'agit de s'interroger ici sur l'originalité du rapport des Égyptiens avec cet élément de premier plan dans la topographie égyptienne. Pour cela, nous ferons appel à plusieurs niveaux de lecture. Les différents usages du sable, à la fois corporels, domestiques et techniques, seront tout d'abord exposés. Seront ainsi questionnées les sources qui mettent en lumière 


\section{Graphies hiéroglyphiques des différents termes évoquant le sable}

1a: « sable » (châou), la graphie se compose de trois signes hiéroglyphiques, deux phonogrammes (le bassin et le bras), et un déterminatif (les trois grains de sable). $\mathbf{1 b}$ : « banc de sable »

(tjès), la graphie se compose d'un phonogramme (le nœud), d'un complément phonétique (le verrou) et de deux déterminatifs (la section de terrain irrigué et le trait qui marque l'unité). 1c: « haut-fond » (chaout), la graphie se compose de trois phonogrammes (le fourré de papyrus, le poussin de caille et le pain) et d'un complément phonétique (le vautour percnoptère). $1 \mathrm{~d}$ : « banc de sable » (nétjéset), la graphie se compose de trois phonogrammes (le filet d'eau, le lien pour les animaux et le siège), d'un complément phonétique (le pain) et d'un déterminatif (Tilapia nilotica).
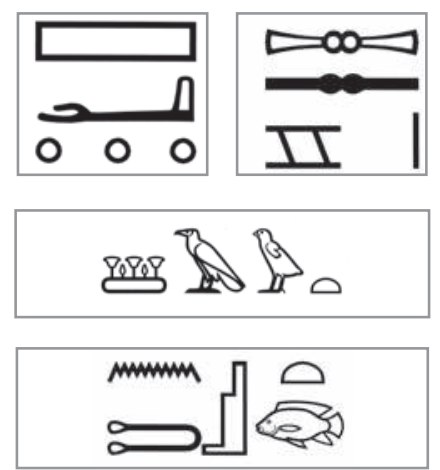

les réalités d'une difficile cohabitation quotidienne, réalités mises en évidence par l'archéologie, mais qui illustrent également un phénomène d'appropriation du sable en tant que matériau, à l'origine de la mise en œuvre de procédés techniques originaux. Dans un second temps, quelques exemples de pratiques rituelles et de mythes faisant intervenir le sable seront examinés afin de répondre à la question de la place du sable dans le discours religieux égyptien, indice d'une manière d'appréhender le sable propre à la culture égyptienne.

\section{Le sable dans le quotidien des Égyptiens}

Image satellite du territoire égyptien actuel

On estime que $96 \%$ de la surface du pays est recouverte de sable. On distingue la teinte verte du Delta et de la Vallée du Nil qui correspond aux terres cultivées.
La particularité du paysage égyptien, marqué par la limite nette entre la vallée fertile et l'espace désertique, n'a cessé d'étonner au cours des siècles et constitue toujours une curiosité pour les visiteurs contemporains. Hérodote (Barguet 1964)² a décrit ainsi le Delta du Nil: « la partie de l'Égypte où abordent les vaisseaux des Grecs est une terre d'alluvions, un don du fleuve, de même que la région qui s'étend à trois jours de navigation en amont du lac ». Pour Diodore de Sicile (Vernière 1993) ${ }^{3}$, « le Nil, par ses bienfaits envers les hommes, surpasse tous les fleuves de la terre habitée». Strabon (Yoyotte et al. 1997) ${ }^{4} a$ finalement résumé ainsi la géographie égyptienne: "l’Égypte se réduit aux terres alluviales, c'est-à-dire à l'extrême bande de terres alluviales s'étendant de part et d'autre du Nil qui, des frontières de l'Éthiopie au sommet du Delta, n'occupe que rarement, d'un seul tenant, une surface habitable d'une largeur de trois cents stades. Ainsi, quand elle est asséchée, ressemble-t-elle par sa longueur à un ruban, si l'on excepte les dérivations les plus importantes du fleuve ». La surface du pays est en effet recouverte à $96 \%$ de sable, le « ruban » de terre fertile qui s'est vu progressivement occupé par les populations n'ayant jamais excédé quelques kilomètres de large. Les Égyptiens étaient donc 
en contact permanent avec le sable désertique qui les environnait. Le vent apportait régulièrement du sable sur les terres habitées, le phénomène étant considérablement accru lors des tempêtes de sable qui étaient susceptibles de s'abattre sur le pays pendant les mois de juin et juillet. S'ajoutait à cela l'avancée progressive du désert. Si les témoignages écrits sur l'habitat sont relativement rares, il est évident que la vie devait être considérablement perturbée par la proximité des installations humaines avec le sable. Nous sommes en revanche un peu plus renseignés sur les problèmes que le sable a pu poser dans l'agriculture, en particulier pour les périodes les plus récentes de l'histoire égyptienne. Les documents juridiques ptolémaïques (III ${ }^{\mathrm{e}} \mathrm{I}^{\mathrm{er}} \mathrm{S}$. av. J.-C.) font ainsi état de clauses prévoyant la contamination d'une terre par le sable déposé par une crue trop abondante, rendue impropre à la culture (Grenfell, Hunt 1901 : 106) ${ }^{5}$. Une terre ensablée était déduite des impôts à payer, puis travaillée pour pouvoir être remise en culture. L'existence même d'une clause de ce genre atteste le caractère banal du fait.

Les parcelles agricoles étant séparées par du sable, celui-ci pouvait être facilement déposé sur les champs par le vent. Outre le terrain cultivé, le sable envahissait également les canaux d'irrigation, outils indispensables sur un territoire où la maîtrise de l'eau était vitale. Il fallait curer ces canaux chaque année, au moment de l'étiage. On en retirait alors un mélange de sable et de limon dont on faisait ensuite usage pour consolider les digues existantes. Au même titre que les canaux d'irrigation, ces digues devaient être régulièrement entretenues sous peine de s'effondrer. Les différents travaux d'entretien du domaine agricole incombaient à l'ensemble de la population égyptienne sous forme d'impôt payé en nature. Tout défunt était également amené à s'acquitter de ces tâches dans l'au-delà, domaine du dieu Osiris, où il aspirait à une nouvelle vie. Cette seconde existence ressemblait fortement à celle qu'il avait vécue sur terre. Il y disposait d'un lopin de terre à cultiver qui lui fournissait sa subsistance et il lui fallait donc s'adonner aux travaux des champs. En prévision de cette activité peu enviée, le défunt prenait la précaution de se munir d'ouchebtis, petites figurines funéraires, serviteurs destinés à le remplacer dans les travaux difficiles qui l'attendaient. Le texte (Barguet $1967: 42)^{6}$ dont les ouchebtis sont marqués explique qu'ils avaient, parmi toutes sortes de tâches telles que celles de travailler la terre ou de l'irriguer, à transporter du sable, probablement le sable qui, comme nous l'avons expliqué, envahissait progressivement les champs et dont il fallait se débarrasser.

Toutefois, le rôle du sable dans l'agriculture met particulièrement bien en évidence son caractère ambivalent. En effet, lorsqu'il s'agit d'agriculture égyptienne, il faut distinguer d'une part sable alluvial et d'autre part sable désertique. Le sable alluvial, mélangé à d'autres substances, pouvait être utilisé pour fertiliser les champs alors que le sable désertique constituait un problème dans le développement même de l'agriculture, difficulté que les Égyptiens ont réussi à surmonter en mettant en œuvre des parades. En 256 av. J.-C., sous le règne de Ptolémée Philadelphe, un personnage du nom de Zénon part s'installer dans la région du Fayoum, oasis de l'Ouest égyptien, avec pour mission de remettre en valeur certains territoires abandonnés au désert (Orrieux 1983 : 77, 82). Irrigués et plantés en espèces adaptées à ce type de sol, ils deviennent des territoires agricoles, symboles de la victoire des Égyptiens contre l'envahissement progressif de leur terre par le sable.

L'ensablement concernait également les monuments ainsi que l'illustrent plusieurs sources écrites. C'est le cas par exemple du célèbre sphinx de Gîza, réalisé à la IVe dynastie (env. 2600-2475 av. J.-C.) et presque entièrement enseveli sous le sable du désert plus de mille ans après, alors que le jeune Thoutmosis IV était encore prince. Un texte, inscrit

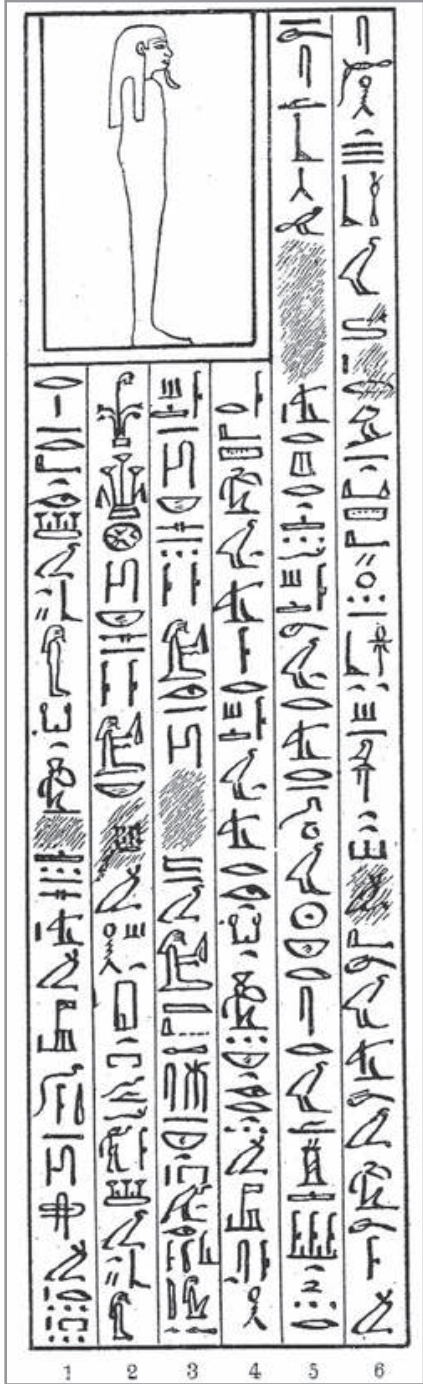

\section{Chapitre VI du Livre des Morts (version du Papyrus de Nebseni, XVIII dynastie)}

Le texte évoque les tâches confiées aux ouchebtis, serviteurs du défunt dans l'au-delà: « on te désignera pour moi (à ma place) à tout moment pour rendre cultivables les champs, pour inonder les rives et pour transporter le sable de l'Orient à l'Occident ». (E. Naville, 1886, vol. I, pl. VIII). 
Stèle dite du songe, Gìza, Thoutmosis IV (1397-1387 av. J.-C.)

Conservée entre les pattes du célèbre sphinx, la stèle décrit le rêve au cours duquel le sphinx s'adresse au prince pour lui demander de procéder à son désensablement. sur une stèle qui a conservé son emplacement d'origine, sur le plateau de Gîza, entre les pattes antérieures de l'animal, met en scène le prince Thoutmosis, endormi à l'ombre du monument après une partie de chasse. Le sphinx s'adresse à Thoutmosis en songe et lui demande d'intervenir en sa faveur en dégageant le sable qui le recouvre presque complètement: « Le sable du désert sur lequel je me dresse se rapproche de moi. Aussi me suis-je hâté de te confier la réalisation de ce qui est dans mon cœur; car je sais que tu es mon fils, mon protecteur. » (Zivie 1976 : 131)

Les monuments de culte devaient être régulièrement entretenus, sous peine d'être ensevelis. Pendant les moments de troubles politiques ou en cas d'abandon, la nature reprenait vite ses droits, le sable envahissant et masquant rapidement les constructions. C'est ainsi que la reine Hatchepsout (XVIII dynastie, 1479-1458 av.J.-C.), dans un texte du temple du Spéos Artémidos, en Moyenne-Égypte, rapporte qu'elle a fait dégager le temple d'Hathor de Cusae, alors laissé à l'abandon et par conséquent recouvert de terre et de végétation, mais également rempli de sable (Allen 2002).

Les tombes et leurs chapelles, destinées à abriter le culte rendu aux défunts par les membres de leur famille, voire par les services d'un clergé spécifique, subissaient aussi les assauts du sable, d'autant plus qu'elles étaient implantées à la lisière du désert. Pour protéger ces espaces, on a ainsi pu construire des murs destinés à arrêter le sable afin de l'empêcher de pénétrer dans les cours des tombes (O'Connor 1985).

Le sable représentait également un obstacle dans les déplacements quotidiens. On imagine sans peine le travail fastidieux que devait représenter le dégagement régulier des

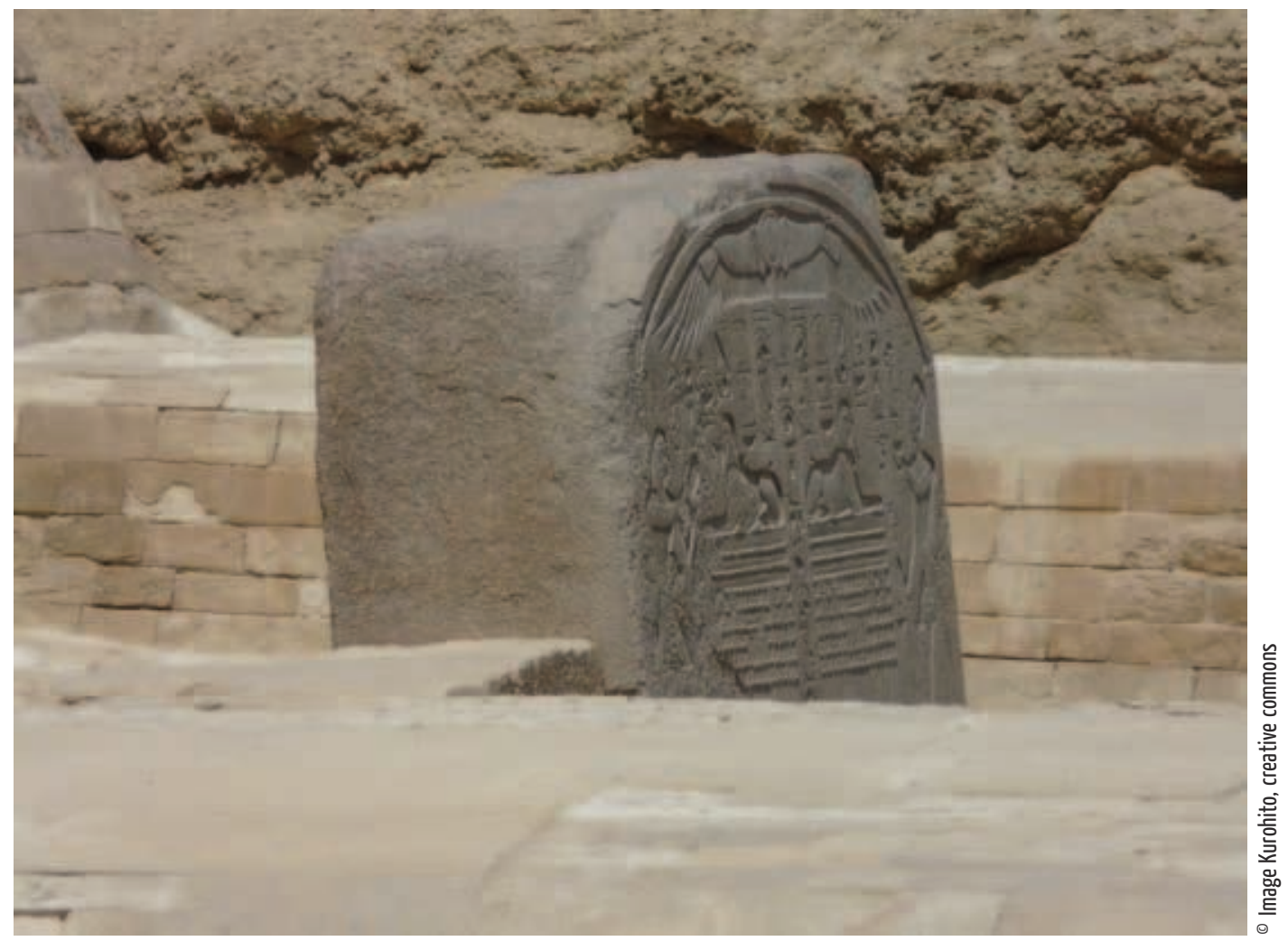


pistes ou des voies de circulation, probablement recouvertes de sable en quelques jours, même quelques heures, lors des épisodes de tempêtes. Les Égyptiens ont fait preuve d'ingéniosité pour s'adapter à leur environnement en développant des modes de transport appropriés. Dans les tombes de l'Ancien Empire (env. 2670-2195 av. J.-C.), il n'est pas rare de rencontrer des scènes de halage de sarcophages ou de statues de taille imposante. On y voit généralement plusieurs personnages tirer le traîneau sur lequel repose le sarcophage ou la statue, précédés de l'un de leurs compagnons versant de l'eau. Avant d'humidifier le sol, il semblerait que ce dernier y ait déposé une couche de limon: l'argile mouillée contenue dans ce limon, alors rendue particulièrement glissante, facilitait grandement la manœuvre. Cette technique a été appliquée pour tous les transports de charges.

Enfin, il ne faut pas oublier le déplacement par voie d'eau puisque le sable constituait une contrainte majeure pour la navigation. Le Nil n'était praticable qu'une partie de l'année, le niveau de l'eau interdisant toute navigation pendant l'étiage. Le cours du fleuve était capricieux et les hauts-fonds et bancs de sable spécialement redoutés des navigateurs, les accidents ne devaient pas être rares. Des textes officiels, récits d'expéditions royales vers les régions du Sud, témoignent ainsi des difficultés rencontrées. Le convoi devait parfois s'arrêter sous peine de s'échouer sur des bancs de sable, formations naturelles constituées de sable alluvial. Il fallait alors descendre de l'embarcation afin de passer l'obstacle pour pouvoir continuer le voyage (Dunham 1967 : 33-34).

Une autre thématique importante dans la sphère quotidienne est celle du rapport du corps humain au sable. En effet, comme toutes les populations en contact avec les zones désertiques, les Égyptiens ont eu à souffrir physiquement de la cohabitation avec le sable ainsi qu'en témoignent les momies retrouvées sur le sol égyptien. Les effets les plus néfastes pour la santé étaient dus à l'inhalation chronique de sable, à l'origine de graves infections pulmonaires. L'examen des poumons d'une momie découverte par une équipe américaine montre ainsi un taux de silice dans les poumons supérieur à la normale (Reeves 1992 : $34)^{7}$. On parle dans ce cas de pneumoconiose, maladie provoquée par une rétention de poussière dans les poumons (Nicholson \& Shaw 2000 : 382). Elle peut avoir un caractère bénin, mais se révèle maligne si la poussière est siliceuse, engendrant alors une silicose. Cette accumulation de poussière dans les poumons trouve son origine dans une exposition répétée à l'atmosphère désertique, couplée à l'action des tempêtes de sable. Une étude menée sur une population de Bédouins du désert du Néguev (Bar-Ziv \& Goldberg 1974) a montré que les femmes étaient les plus touchées par le phénomène, d'une part parce qu'elles exercent, dès le plus jeune âge, des activités à l'extérieur, d'autre part parce que ces activités s'accompagnent d'une position assise ou accroupie qui favorise l'inhalation de poussière désertique. Il faut également noter que le port du voile ne les protège en rien. Nous ne sommes pas renseignés sur les protections vestimentaires utilisées par les Anciens Égyptiens lorsqu'ils étaient amenés à s’aventurer dans les zones désertiques ou pendant les périodes de tempêtes de sable. Quoi qu'il en soit, si protection il y a eu, elle ne s'est pas révélée très efficace au vu de la quantité de silice généralement retrouvée dans les poumons des momies étudiées au cours des dernières décennies (David 2011 : 279-280) ${ }^{8}$. De plus, il semblerait que ces affections respiratoires aient touché, tout au long de l'histoire égyptienne, toutes les catégories de la population sans distinction de rang social. Si l'on peut penser que certaines personnes devaient être plus particulièrement exposées, selon leur profession ou leur mode de vie, les dangers que le sable représentait pour la santé étaient cependant l'affaire de tous.

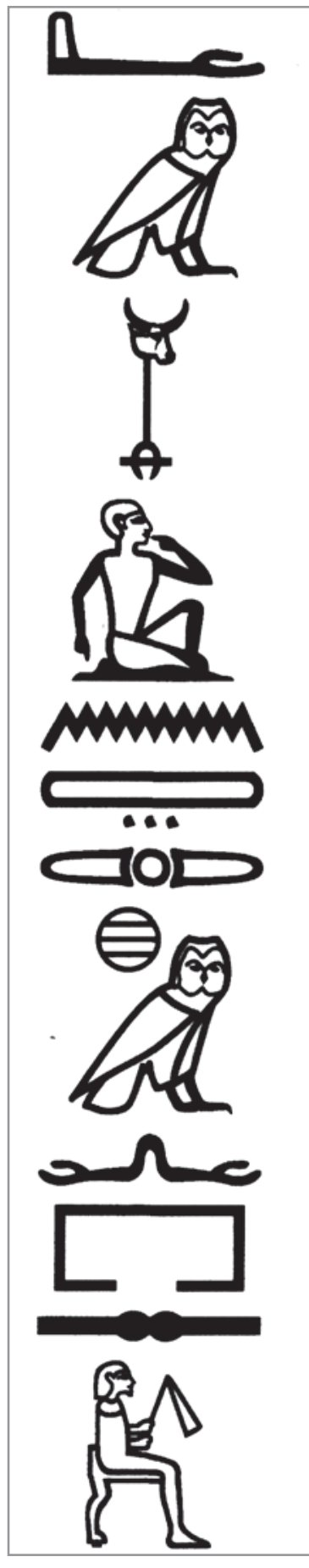

Texte du Spéos Artémidos, Hatchepsout (1479-1458 av. J.-C.)

II détaille l'état du temple de Cusae, laissé à l'abandon: « la terre avait avalé son noble sanctuaire ». 


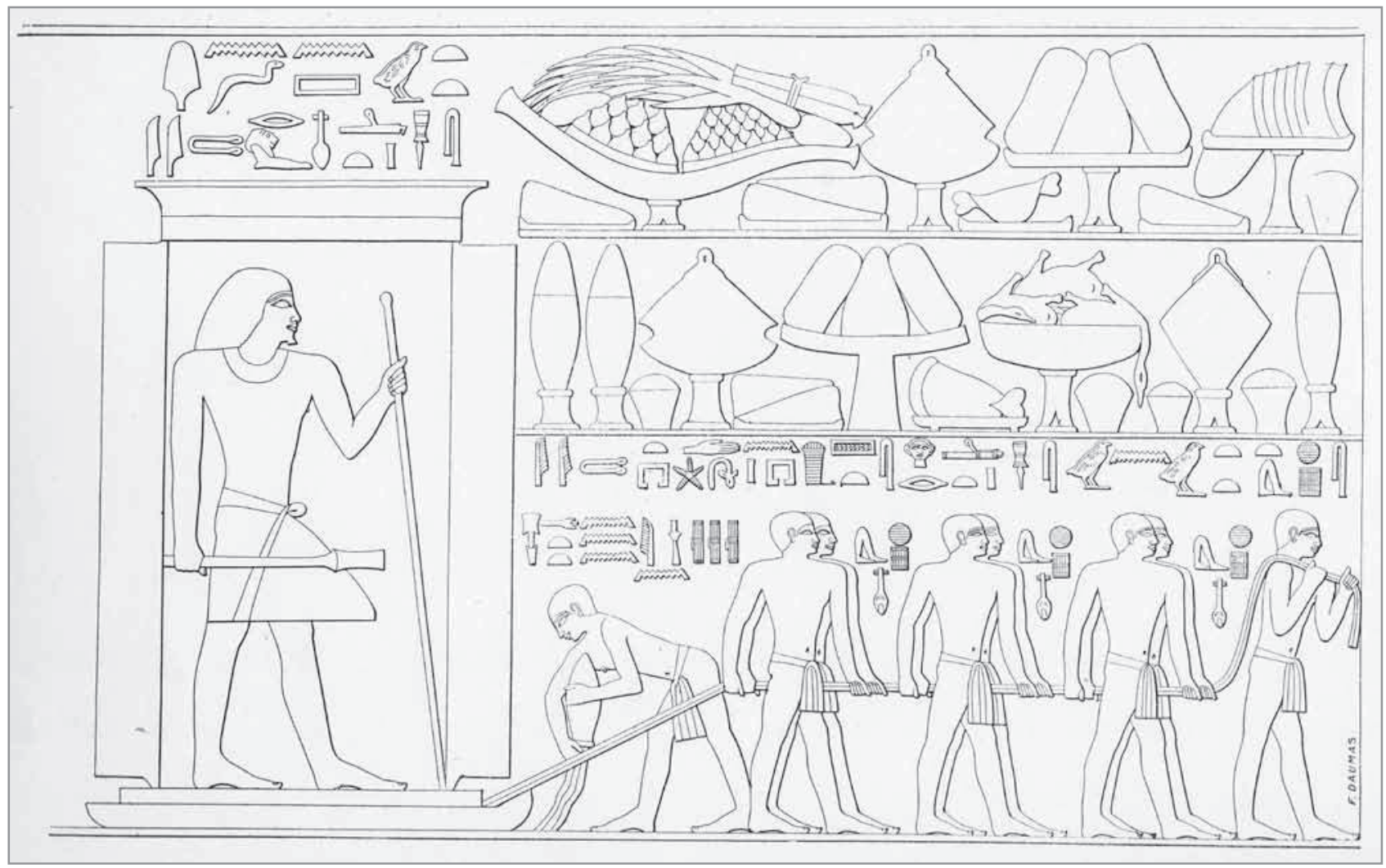

Scène de halage d'une statue dans le tombeau de Ti, Saqqarâ, Ve dynastie

(L. Épron, Fr. Daumas, G. Goyon 1939, pl. LII).
La remarque est également valable pour ce qui concerne la dentition des Anciens Égyptiens. Les mâchoires des corps mis au jour montrent très souvent une forte abrasion des dents, à l'origine de la formation d'abcès, de kystes, voire d'une modification du mouvement de la mâchoire (Reeves 1992 : 17). La faute en est en grande partie imputable au régime alimentaire des Égyptiens, fondé sur le pain. Ce pain comptait un fort taux de silice, à la fois à cause de la pollution des champs de céréales par le sable mais aussi de la mauvaise qualité des meules qui se désagrégeaient peu à peu (Nicholson \& Shaw 2000 : 379, 565). Le phénomène a donc touché toutes les catégories de la population, des marques d'usure ayant même été constatées sur les dents du pharaon Ramsès II (Ruffer 1921 : 287).

Le sable s'insinuant partout, les Égyptiens avaient aussi à redouter les maladies oculaires. Pour s'en prémunir, ils ont développé des khôls et des poudres, dont une élaborée à partir d'un mélange de sable, de natron et de minéraux de cuivre (Reeves 1992 : 58). En effet, si le sable a pu influer sur la santé des Égyptiens, il a paradoxalement participé à ce que l'on pourrait appeler les « soins du corps », qu'il s'agisse du corps vivant ou du cadavre. Le sable entrait par exemple dans la composition de certains baumes appliqués en cas d'affection de la peau ou de morsure de serpent venimeux (Bardinet 1995 : 353, 530). Les Égyptiens l'employaient également comme substitut de savon pour la toilette en association avec du natron dilué, pratique encore familière aux populations sahariennes contemporaines.

Le sable a eu aussi un rôle à jouer dans le traitement du cadavre. Ce dernier se conserve naturellement dans l'atmosphère désertique, si bien que l'on a pu penser que la mise en 
place du processus de la momification n'était pas étrangère à une longue observation du sort réservé aux dépouilles abandonnées au désert. Les Égyptiens les plus humbles ont toujours été enterrés dans une fosse à même le sable, sans traitement particulier. Il n'est pas rare de retrouver aujourd'hui ces corps desséchés par le sable, généralement en bon état de conservation. Matériau abondant et facile à obtenir, du sable grossier était parfois utilisé pour remplir des sacs de toile déposés dans la cavité abdominale à la place des viscères, ôtés au cours de l'embaumement.

À côté de tous les désagréments dont il était la source, le sable était envisagé comme un matériau, c'est-à-dire un élément naturel dont des propriétés remarquables ont été mises à profit. Les textes égyptiens évoquent ainsi un certain nombre de savoir-faire élaborés à partir du sable, pratiques que l'archéologie parvient parfois à mettre en évidence. Le sable était tout d'abord employé dans l'artisanat, ce qui se comprend lorsqu'on songe à son abondance et à sa facilité d'accès. Les Égyptiens l'utilisaient par exemple comme dégraissant dans la pâte pour la céramique ou dans la pâte à stuc, la teneur en sable variant suivant la qualité de cette pâte. Il pouvait également entrer dans la composition des mortiers en tant que charge granulaire. Les propriétés abrasives du sable étaient recherchées dans la menuiserie. Le procédé est illustré par une scène de mastaba de l'Ancien Empire: une couche d'un mélange de sable et d'eau était déposée sur un ouvrage de bois que l'on frottait avec un ustensile rond, probablement un caillou, pour en polir la surface. La même technique servait au polissage des statues et au façonnement des parements (Goyon \& Golvin 2004 : 70, 75; Nicholson \& Shaw 2000 : 66). Mis à part les figurines osiriennes, dont nous reparlerons plus loin, aucun objet façonné en sable n'a été retrouvé, ce qui ne constitue pas la preuve qu'il n'en existait pas. Ainsi, certains textes indiquent que des figurines en cire, destinées à un usage unique, étaient fabriquées dans le cadre de rituels magiques. Peut-être existait-il des objets similaires, faits de sable. Particulièrement fragiles, ils n'ont probablement pas été conservés.

Les Égyptiens faisaient différents usages du sable dans l'architecture. Il est très vraisemblable qu'ils employaient le système du caisson à sable pour dresser les obélisques, destinés à orner les grands temples. Acheminé par bateau depuis les carrières, l'obélisque était placé à l'horizontale sur une rampe en brique crue, environnée d'échafaudages de même hauteur, et dont au moins un des côtés était en pente douce. Les deux parois de la rampe enfermaient un caisson de sable sec. La partie basse de l'obélisque, amenée à reposer sur un socle déjà en place au fond du caisson, était disposée de manière à pénétrer en premier dans le caisson. Par un système d'écoulement à la base du caisson, le sable était peu à peu retiré, entraînant lentement et de façon régulière l'obélisque à basculer. Suivant la tournure que prenaient les événements, l'écoulement du sable pouvait être accéléré, ralenti ou tout simplement arrêté. Une fois le caisson complètement vidé, l'obélisque se retrouvait appuyé contre la rampe de terre crue, une arête déjà en place sur le socle. Il ne restait alors qu'à le tirer pour qu'il prenne sa position verticale définitive et repose complètement sur sa base (Goyon \& al. 2004 : 331-333). On pense qu'une procédure du même type était appliquée pour mettre en place les colosses devant les temples.

En outre, la technique du puits de sable est attestée dans certaines tombes, dès la XII ${ }^{e}$ dynastie (1994-1781 av. J.-C.). Elle permettait de manier le sarcophage en pierre, extrêmement lourd, juste avant que

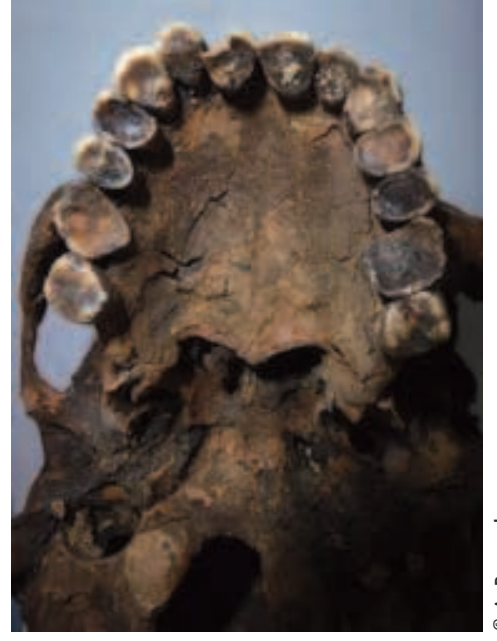

胥

Traces d'attrition sur un maxillaire de momie égyptienne

Momie 0xy 2010-22713. Nécropole d'Oxyrhynchos (Moyenne Égypte).

Scène de menuiserie, polissage du bois dans le tombeau d'Oupemnéfret, Gîza, Ve dynastie «Polir le sarcophage. Mettre l'eau, mettre le sable. »

(S. Hassan 1936, fig. 219).

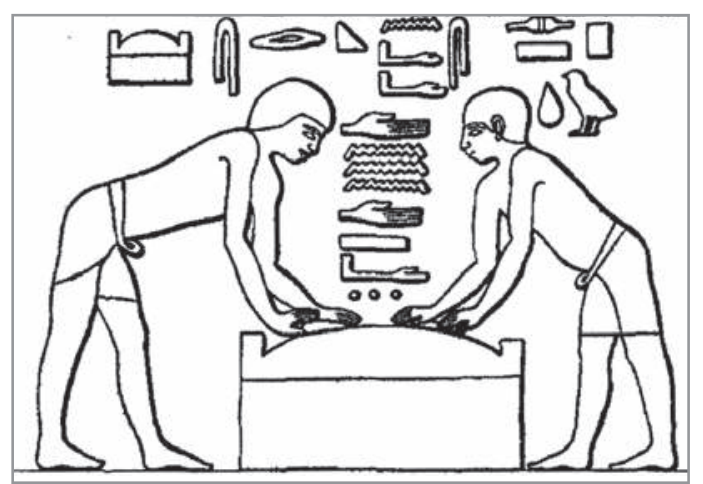


Hypothèse d'élévation d'un obélisque

(Dessin J. Misuriello

d'après J.-Cl.Goyon, J.-Cl.Golvin,

Cl. Simon-Boidot, G. Martinet 2004, fig. 431a).

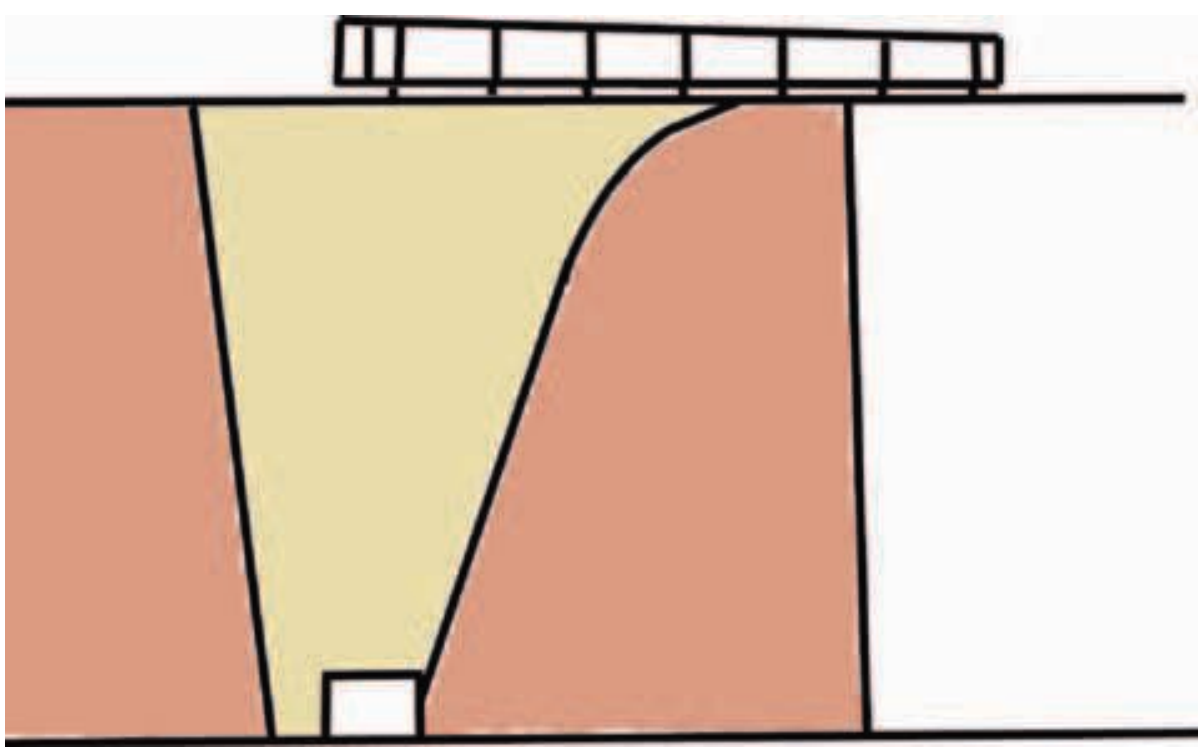

la tombe ne soit fermée: par un système de puits connectés les uns aux autres, on retirait le sable placé sous le sarcophage qui venait alors prendre sa place définitive (Arnold 1991 : 74-75). Le sable intervenait plus directement dans la construction d'édifices puisqu'il constituait la plupart du temps l'assise de fondation. Nous aurons l'occasion de revenir sur ce point lorsque nous évoquerons le rituel de fondation du temple égyptien.

On retiendra donc le caractère duel, ambivalent, du sable dans les différents aspects de la vie quotidienne, puisqu'il se révèle être à la fois une contrainte, un élément redouté et envahissant contre lequel la lutte est permanente, et un matériau dont les qualités exceptionnelles ont été mises à profit dans plusieurs domaines. On en vient alors à questionner la place que tient le sable, élément omniprésent dans le paysage et par conséquent dans le quotidien, dans le discours religieux égyptien dont on sait qu'il s'inspire fortement de l'observation de la nature.

\section{Le sable dans le discours religieux égyptien}

Il est intéressant d'observer que dans les différents rituels dans lesquels on le mobilise, le sable semble rattaché à des valeurs qui font écho à l'ambiguité qui le caractérise au quotidien, élément bénéfique par certains aspects mais aussi néfaste par d'autres.

Ainsi, dans certains contextes, le sable semble clairement associé à des valeurs positives qui peuvent être résumées en cinq termes: pureté, sacralité, renaissance, protection et fertilité.

Une première caractéristique du sable est son lien avec la mort. C'est en effet à la limite entre désert et terres cultivées qu'étaient implantées les nécropoles, à la fois pour des raisons pratiques et symboliques. Sokar, un dieu chthonien très ancien associé à la nécropole de Memphis, porte notamment l'épithète « Celui qui est sur son sable » (Leitz et al. 2002 : 671-672), c'est-à-dire probablement « Celui qui domine son sable ». Sokar 
est figuré sous la forme d'un faucon, animal qui survole son territoire. Dans ce contexte funéraire, le sable revêt un caractère protecteur vis-à-vis de la tombe. Accumulé sous la forme d'une butte, il protège le corps du défunt, qu'il s'agisse d'un homme ou d'un dieu. Certains textes précisent ainsi que le sable qui recouvre la tombe des dieux primordiaux,

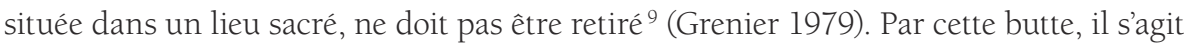
probablement de matérialiser l'emplacement d'un lieu de culte important. Dans le cas de l'homme défunt, on peut penser que le sable vient au contraire masquer l'entrée de la tombe aux yeux des pillards tout en signalant son emplacement sous la forme d'un tertre.

On rencontre également à plusieurs reprises le sable en tant qu'élément purificateur et sacralisant. C'est le cas par exemple dans le rituel accompli quotidiennement pour la divinité dans chaque temple d'Égypte. Le roi, remplacé en pratique par un prêtre, était censé rendre plusieurs fois par jour un culte à la statue divine, conservée dans le naos du temple. Le rituel, fixé de manière canonique et conservé sur différents supports dont les parois des chapelles du temple de Séthy $\mathrm{I}^{\mathrm{er}}$ à Abydos, comporte de nombreux épisodes. Il se résume ainsi : l'officiant sort la statue divine de son naos, procède à sa toilette, l'habille, la pare de bijoux, la farde et lui présente différentes offrandes alimentaires. Parmi les épisodes qui rythment le rituel figure celui de « verser du sable ». Ce sable, probablement de qualité particulière même si le texte ne le précise pas, semble être versé pour former une station d'accueil à destination de la statue divine que l'officiant doit laver. Placée sur ce sable, qui prend probablement la forme d'une petite butte, la statue divine se trouve dans des conditions optimales, en sécurité, pour recevoir sa toilette.

On retrouve la même idée dans un rituel funéraire accompli sur la momie ou la statue du défunt devant la tombe: le rituel de l'Ouverture de la Bouche. À cette occasion la momie, ou la statue, était placée sur un petit monticule de sable. Ce rituel était destiné à restituer ses facultés sensorielles au défunt en vue de sa nouvelle vie. On ouvrait les oreilles, les yeux, le nez et la bouche de la momie ou de la statue à l'effigie du mort afin de garantir à ce dernier la fonctionnalité de ses sens dans l'au-delà. Dans ce contexte, il semble que le sable soit également investi de la faculté de purifier un espace.

Comme nous l'avons mentionné plus haut, le sable sert d'assise de fondation aux monuments égyptiens. L'action de remplir la tranchée de fondation avec du sable est ritualisée. Sur les représentations du rituel conservées dans certains temples, on voit le roi, officiant par excellence, muni d'un récipient, peut-être un panier, en train d'accomplir ce geste. Le choix du sable répond à plusieurs exigences techniques. Le sable garantit en effet la stabilité de l'édifice par son incompressibilité, permet d'atteindre le niveau horizontal sur lequel bâtir les fondations de l'édifice, ou encore prévient d'éventuelles fissures provoquées par les remontées de la nappe phréatique. Il a également une fonction anti-sismique (Zignani 2008 : 124). Cependant, des aspects religieux sont également à prendre en compte: il s'agit ici de purifier l'espace, en l'occurrence l'assise du temple, et d'assurer ainsi la pérennité de l'édifice, mais peut-être aussi de rappeler la création du monde. Les textes jouent sur cette idée en indiquant que la tranchée est creusée « jusqu’à l'eau », c'est-à-dire jusqu'à la nappe phréatique, puis « comblée de sable ». Le sable déposé en quantité dans la tranchée sur cette eau est alors probablement interprété comme une évocation de la butte primordiale faite de sable émergeant du milieu liquide originel que constituait le Noun, épisode fondateur rapporté par certaines cosmogonies (Zandee 1947: pl. II). Cette image de la butte surgissant du Noun puise certainement son origine dans l'observation directe de la création des geziras, petites îles formées par les mouvements du Nil provoquant des dépôts d'alluvions, et caractérisées par un sol limoneux fertile. Ainsi

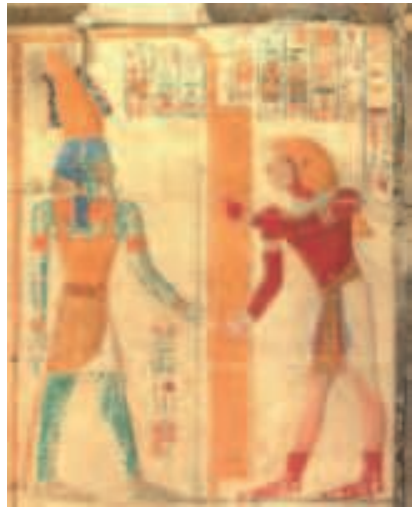

\section{Scène du Rituel du culte divin journalier, représenté dans le temple de Séthy ler à Abydos}

Le roi, officiant par excellence, ouvre les portes du naos enfermant la statue d'Osiris. Composé d'un grand nombre d'épisodes, ce rituel était théoriquement célébré quotidiennement afin de servir la divinité tutélaire de chaque temple. (Calverley, Broome 1933, pl. 1). 


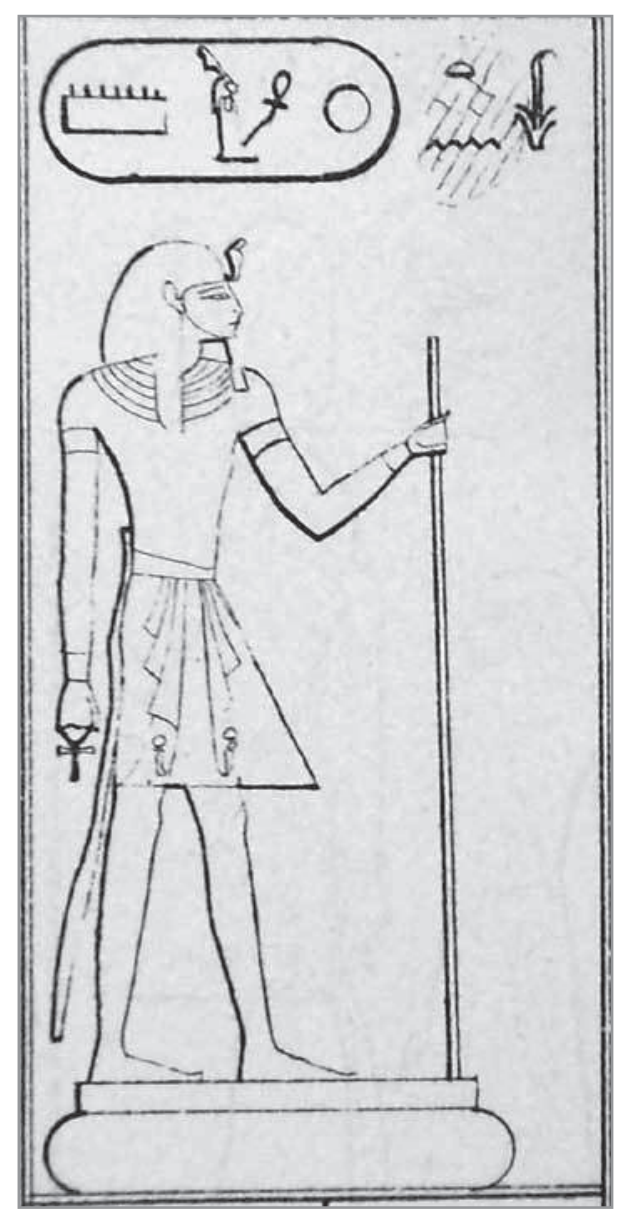

Statue du défunt placée sur un coussin de sable au cours du rituel de l'Ouverture de la Bouche, scène de la tombe de Séthy ler (KV 17), Thèbes, XIXe dynastie (Lefébure 1886, pl. XLIII). les Égyptiens ont-ils tenté d'expliquer la création du monde d'après ce dont ils étaient témoins quotidiennement. En effet, la religion égyptienne se nourrit de l'observation naturaliste: les phénomènes naturels sont observés et intégrés au discours mythique dans une tentative d'explication du fonctionnement du monde. Cette butte qui sert de support au créateur abrite la première forme de vie. Elle est aussi associée à la fertilité. Or on rencontre le sable comme ingrédient des figurines fabriquées à l'image du dieu Osiris lors de ses festivités annuelles (Cauville et al. 1997). Osiris, divinité funéraire et souverain du royaume des morts, est également celui qui a initié les hommes à l'agriculture. Sa mythologie, en relation avec le cycle des saisons, est liée à la crue du Nil. Au cours de ces fêtes osiriennes célébrant le retour de la végétation, on moulait donc de petites figurines à l'effigie de la divinité. Du sable alluvial, probablement choisi pour son caractère fertile, entrait dans leur composition, mélangé à de la terre, des graines et des pierres précieuses. Ces figurines étaient arrosées plusieurs fois par jour jusqu'à germination des graines manifestant à la fois le retour de la végétation et la renaissance du dieu.

Si certains rituels illustrent des aspects bénéfiques du sable, d'autres mettent au contraire en relief des valeurs négatives. Son usage peut être préconisé contre les adversaires, pour les aveugler par exemple (Ritner 1993 : 156-157). Une place sableuse est parfois mise en relation avec leur châtiment (Darnell 2004, pl.33). Le sable est alors synonyme de dangerosité, de léthargie, d'hostilité voire d'infertilité ou de stérilité.

Trois formules funéraires ${ }^{10}$ insistent notamment sur le fait que le défunt ne doit pas avoir le visage recouvert de sable. Si le sable de la butte funéraire est protecteur, on semble redouter le contact du corps avec le sable. Le sable, sorte de chape menaçant de peser sur le mort, est ici associé à un état de léthargie, à un sommeil profond et irréversible duquel le défunt risque de ne jamais sortir. Cette inertie est véritablement l'une des craintes majeures du mort égyptien, appelé à se mettre en mouvement pour rejoindre l'autre monde. Nous avons vu que le sable (la silice) peut être à l'origine de maladies respiratoires. Les textes sont clairs: c'est bien le visage du défunt qui ne doit pas être recouvert. Il est tentant de voir dans cette précision une référence à une préoccupation liée au monde des vivants: le sable envisagé comme une menace pour l'intégrité physique, principalement au niveau respiratoire.

En outre, du sable est impliqué dans des événements mythiques dramatiques, telles les luttes du dieu solaire contre ses nombreux ennemis. C'est par exemple sur du sable que se déroule le premier combat opposant le créateur à ses ennemis cosmiques. Le sable en restera marqué comme lieu d'affrontement, sorte d'arène primordiale, trait également développé par l'intermédiaire du banc de sable du serpent Apophis, parangon de l'ennemi et manifestation des forces du chaos concomitantes à la création du monde. Dans la vision égyptienne du fonctionnement du monde, le dieu solaire disparaît le soir à l'ouest, avalé par la déesse céleste Nout qui le remet au monde le matin, à l'est. Pendant la nuit, il voyage dans le corps de la déesse, sur un fleuve. Au cours de sa navigation, il rencontre toutes sortes de créatures du monde souterrain, plus ou moins dangereuses. La plus virulente d'entre elles est probablement Apophis, dont le but est d'arrêter, ou au moins de retarder la traversée du soleil, risque majeur pour la marche 
de l'ordre cosmique. En effet, le soleil, perturbé dans sa course nocturne, pourrait ne pas se lever et laisser le monde dans l'obscurité.

Les textes expliquent qu'afin de mettre son plan à exécution, Apophis commence par avaler l'eau du fleuve sur lequel navigue la barque solaire (Barguet 1967 : 142) ${ }^{11}$. En abaissant le niveau de l'eau, il crée des bancs de sable sur lesquels la barque risque de s'échouer. Pour assurer son succès, le serpent n'hésite pas à venir se placer directement en travers du chemin de l'embarcation comme l'illustrent les tableaux des compositions funéraires inscrites dans les tombes royales à partir de la XVIII e dynastie (dès 1500 av. J.-C.). Le dos d'Apophis est identifié à un banc de sable par le biais d'un jeu de mots, puisqu'en égyptien le terme ts peut désigner la vertèbre, mais aussi le banc de sable. Du point de vue mythologique, le banc de sable constitue ainsi le pendant négatif de l'éminence primordiale sableuse, le schéma binaire de la perception du sable étant respecté.

Il ressort donc très nettement qu'en contexte religieux le sable répond à une double perception, miroir de la situation constatée dans la vie quotidienne. Les préoccupations liées au sable sont transposées dans le monde des dieux et guident la connotation qui est donnée à l'élément.

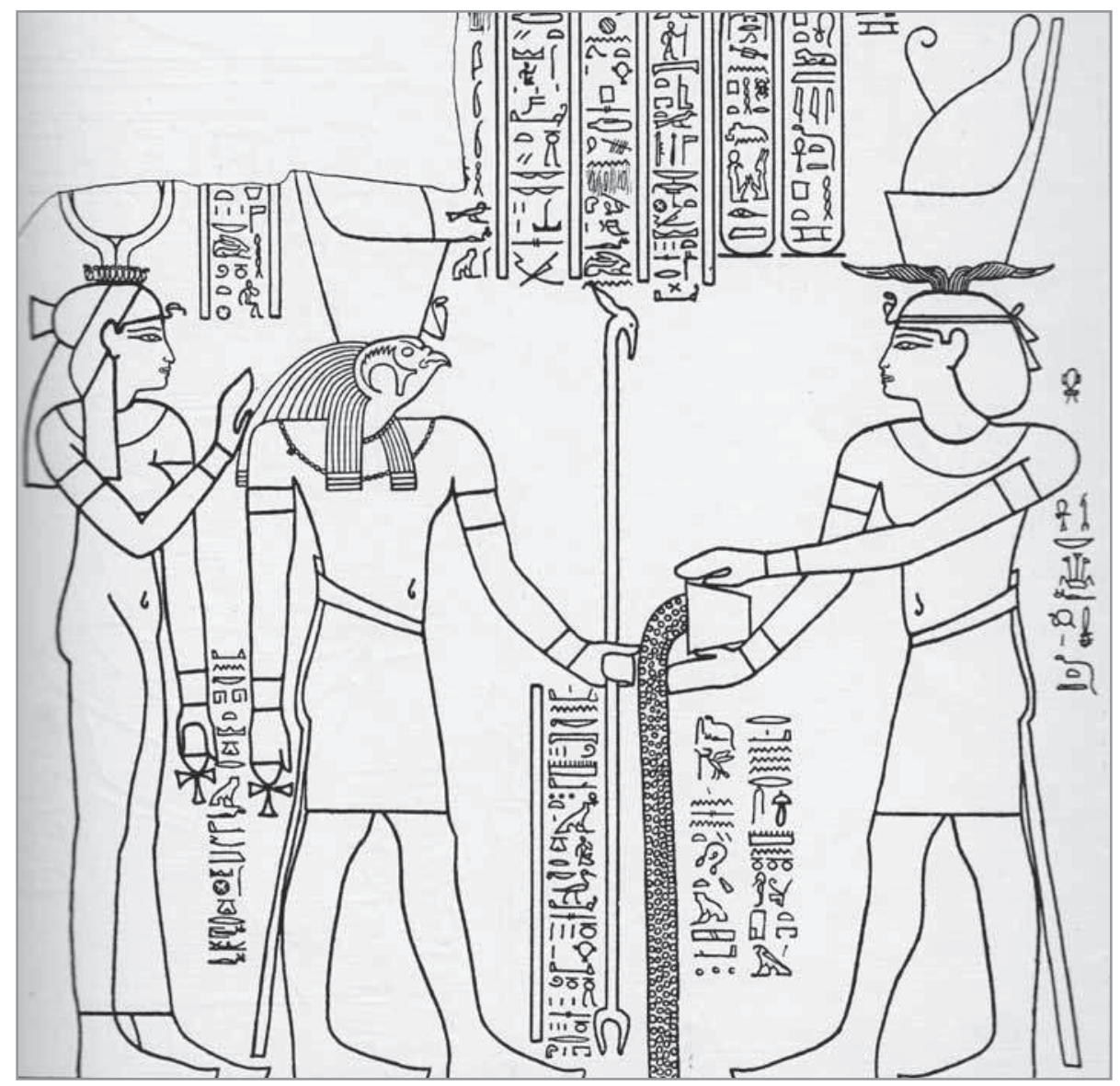

Le roi remplit la tranchée de fondation de sable au cours du rituel de fondation du temple de Kom Ombo

(J. De Morgan 1909, p. 65, n611). 


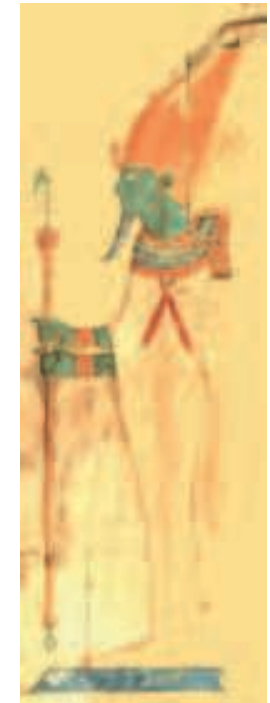

Le dieu Osiris, inventeur de l'agriculture et souverain du royaume des morts, représenté dans le temple de Séthy ler à Abydos

La teinte verte de sa peau est synonyme de fertilité. (Image retouchée d’après Calverley et Broome 1933, pl. 14).

\section{Un statut particulier pour le sable?}

Solide, liquide et meuble, le sable est un élément naturel qui se distingue par des propriétés physiques particulières que les Anciens Égyptiens ont mises à profit. Pour tout ce qui concerne le traitement du corps, qu'il soit vivant ou mort, les Égyptiens ont probablement recherché l'aspect dessiccatif du sable, mais également sa faculté d'abrasion. Son usage dans l'artisanat peut s'expliquer par les mêmes raisons: qualités abrasive et absorbante. Son incompressibilité, le fait qu'il absorbe les éventuelles remontées d'eau, qu'il soit un répartiteur de charges ou encore qu'il permette de créer facilement l'horizontalité sont certainement à l'origine de son utilisation dans la construction. Il faut aussi considérer que le sable commun, grossier, était un matériau abondant et facile d'accès, même s'il semble que dans certains cas précis l'on ait recherché un sable d'une qualité particulière, que l'on n'aura pas hésité à transporter sur des kilomètres (Hikade et al. 2008 : 163).

La prise de conscience des qualités naturelles du sable et leur appropriation nous amènent à considérer que les Égyptiens ont pu attribuer un statut spécifique à cet élément omniprésent dans leur quotidien. L'affirmer reste toutefois délicat puisqu'aucun document n'aborde clairement la question. On trouve par exemple mention du sable dans l'Onomasticon d'Aménémopé, texte présentant des listes de mots classés en catégories, témoin d'une certaine vision du monde (Gardiner 1947) ${ }^{12}$. Le sable y est cité parmi les éléments relatifs à la terre, comme l'argile ou le bois. Il semble que l'on ait voulu faire référence ici au sable alluvial puisque le terme figure juste avant ceux désignant les îles nouvellement formées par le fleuve, c'est-à-dire tout ce qui renvoie aux espaces nilotiques associés au sable fertile. Ce texte ne nous renseigne malheureusement pas beaucoup plus sur la place accordée au sable au sein des éléments de la création.

Certains indices, comme l'intégration du sable au discours religieux et la création d'expressions, d'images mentales, à partir du terme « sable », constituent toutefois quelques éléments de réponse. Le sable a ainsi été associé à l'idée de multitude, les offrandes étant par exemple souvent décrites comme « aussi nombreuses que le sable de la rive ». L'importance du sable aux yeux des Égyptiens découle aussi de son intervention ponctuelle dans les récits cosmogoniques, qui tendent à en faire un élément impliqué dans la formation du monde.

Des similitudes entre les perceptions de l'eau et du sable peuvent être établies: tout comme aucune divinité n'est réellement attachée au sable, aucune n'est directement identifiée à l'eau. L'eau renvoie toujours au fleuve, et plus précisément à sa crue, puisque la pluie est quasiment inexistante. Par ce biais, elle est associée à des idées positives comme la vie, la fertilité, la croissance de la végétation, la fécondité. Elle est également l'élément de base de la purification, caractéristique commune avec le sable. Elle ne semble connotée négativement dans le discours religieux qu'en ce qui concerne les pluies violentes et autres orages, provoqués par le dieu Seth. Si l'abondance de sable pose généralement problème, inversement, c'est plutôt le manque d'eau, les faibles crues, qui sont un sujet de préoccupation. L'eau ne semble donc pas posséder, aux yeux des Anciens Égyptiens, le caractère ambigu attaché au sable qui constitue finalement toute la particularité de cet élément.

Les habitants de la Vallée du Nil, installés dans un environnement à la géographie singulière, marqué par la prégnance du fleuve elle-même renforcée par la proximité avec le désert, se sont visiblement adaptés au sable, et ont développé un rapport particulier avec cet élément des marges de l'espace habité, même si l'on sait aujourd'hui que les 
Égyptiens ont exploré le désert. S'ils ont su mettre en place des solutions pour lutter contre les inconvénients que le sable pouvait représenter, les Égyptiens ont dépassé cette phase de simple adaptation et sont parvenus à tirer profit du matériau en exploitant ses qualités, comme en témoignent leurs réalisations matérielles. Curieusement, dans les sources textuelles les termes qui qualifient le sable sont assez rares. Aucune indication sur la qualité du sable utilisé, sur sa provenance exacte. La forme sous laquelle on l'emploie n'est que rarement précisée, tout comme sa couleur. Toutefois, les découvertes archéologiques permettent parfois d'apporter les informations que taisent les textes égyptiens. Si l'on sait que la distinction entre sable désertique et sable de la rive existe, l'origine du sable est très rarement spécifiée par les textes.

\section{$\&$}

Au-delà d'un simple usage technique, le sable a fait l'objet d'une préoccupation collective puisqu'il a été intégré non seulement au discours par le biais de métaphores mais également aux pratiques religieuses. De plus, l'ambiguité qui le caractérise, que nous avons par exemple mis en évidence dans le rapport du corps au sable, à la fois danger pour la santé et ingrédient de produits destinés aux « soins du corps », se retrouve dans le discours religieux. Présent dans les mythes, le sable est utilisé dans un certain nombre de rites pour les valeurs positives ou au contraire négatives qu'il véhicule, appréciation qui découle directement de l'expérience quotidienne. Cette particularité témoigne de toute l'originalité de la perception égyptienne du sable, marquée à la fois par la volonté de rendre compte d'une situation ambiguë mais aussi de surmonter cette difficulté par des ressorts intellectuels. Mettre en œuvre un modèle théorique autour du sable permet ainsi de donner l'illusion d'un contrôle sur cet élément par essence indomptable, autant craint que recherché, et de le faire entrer dans le monde de Maât, principe d'ordre et de norme sur lequel est fondée la civilisation égyptienne.
Le serpent Apophis - banc de sable menaçant la barque solaire, $7^{e}$ heure de l'Amdouat, tombe de Thoutmosis III (KV 34), Thèbes, XVIII' dynastie (Lefébure 1886, pl. X).

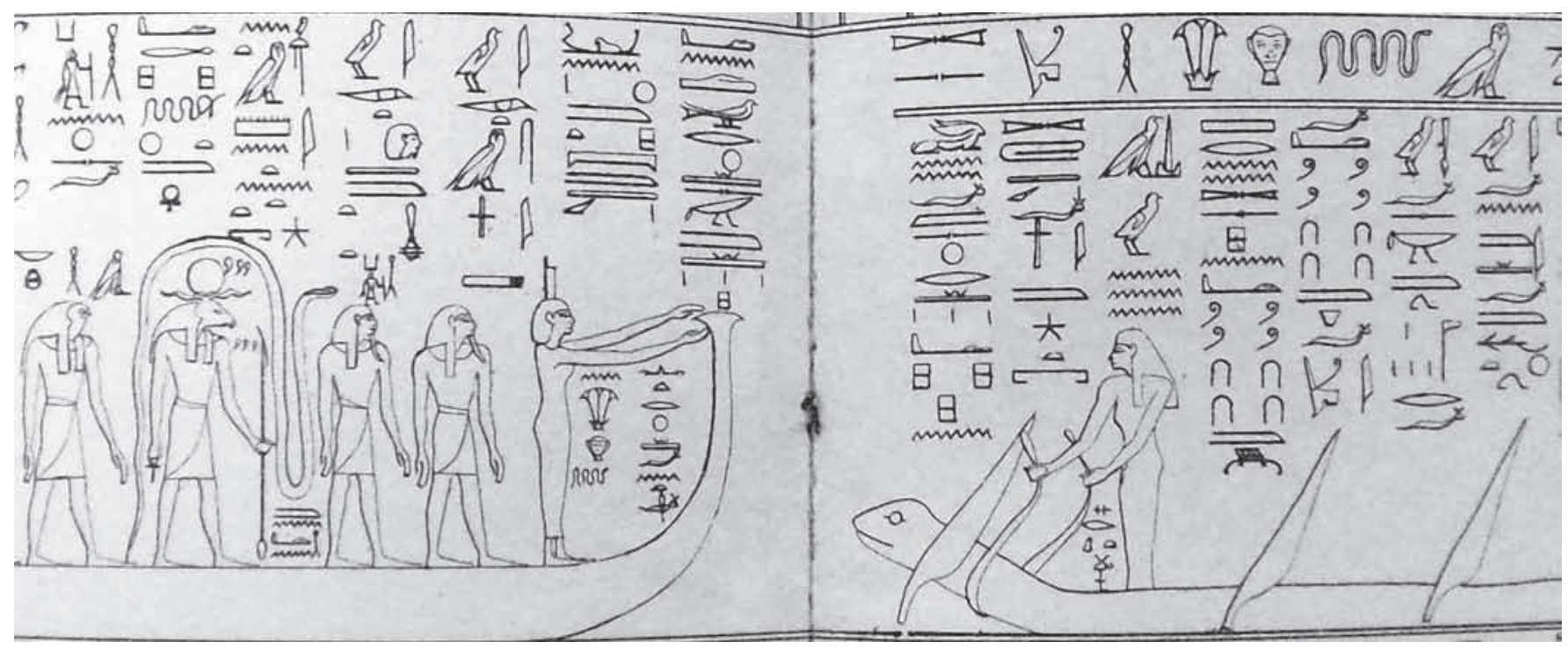




\section{NOTES}

Photo d'ouverture : Saqqarâ Nord, proximité de la pyramide à degrés du pharaon Djoser (III dynastie). Vue sur les immensités sableuses bordant la plus célèbre nécropole d’Égypte ancienne. (Cliché J. Misuriello)

1. Thèse intitulée « Le sable dans les textes mythologiques et rituels de l'Égypte ancienne », préparée sous la direction de M. Bernard Mathieu et soutenue le 26 novembre 2013 à l’Université Montpellier 3 Paul Valéry.

2. L'Enquête, Livre II, 5.

3. Bibliothèque Historique, Livre I, XXVI, 2.

4. Géographie, Livre XVII, I, 4.

5. Papyrus Amh. 85, 15-16.

6. Chapitre VI du Livre des Morts.

7. Momie PUM II (Philadelphie University Museum).

8. Sur le traitement de ces maladies: «Remedies for coughs provided in the papyri include mixtures of honey, acacia and antimony, while respiratory ailments were treated with a plant, Ammi visnaga, which has bronchodilatory properties. »
9. Edfou I, 173, 11-15 et sarcophage de Nes-Ouaiou (NME 6, Medelhavsmuseet de Stockholm).

10. § 1878b [TP 662] (Sethe 1908-1922); formule 23 des Textes des Sarcophages (De Buck 1935-1961); chapitre 169 du Livre des Morts (Naville 1886).

11. Chapitre 108 du Livre des Morts.

12. Document de la XXe dynastie (1185-1069 av. J.-C.). 


\section{RÉFÉRENCES}

Allen, J.-P. 2002 The Speos Artemidos Inscription of Hatshepsut, Bulletin of the Egyptological Seminar 16 : 1-17 et pl. 1-2.

Arnold, D. 1991 Building in Egypt, Pharaonic Stone Masonry. Oxford: Oxford University Press.

Bardinet, Th. 1995 Les Papyrus médicaux de l'Égypte pharaonique. Paris: Fayard.

Barguet, A. 1964 Historiens grecs. I. Hérodote. Thucydide. Hérodote. Paris: Gallimard (Bibliothèque de la Pléiade).

Barguet, P. 1967 Le Livre des Morts des anciens Égyptiens. Paris: Éditions du Cerf (Littératures anciennes du Proche-Orient 1).

Bar-Ziv, J., Goldberg, G. M. 1974 Simple Siliceous Pneumoconiosis in Negev Bedouins, Archives of Environmental Health 29: 121-126.

Buck, A. De 1935-1961 The Egyptian Coffin Texts. Chicago : University of Chicago Press (Oriental Institute Publications 87).

Calverley, A. M., Broome, M. F. 1933 The Temple of King Sethos I at Abydos. Londres, Chicago Press.

Cauville, S., Hallof, J., Van den Berg, H. 1997 Le Temple de Dendara, Les chapelles osiriennes. Le Caire : Institut français d'archéologie orientale (Dendara X/1).

Darnell, J.-C. 2004 The Enigmatic Netherworld Books of the Solar-Osirian Unity, Cryptographic Compositions in the Tombs of Tutankhamun, Ramesses VI and Ramesses IX. Fribourg : Academic Press Fribourg (Orbis Biblicus et Orientalis 198).

David, R. 2011 Ancient Egyptian Medicine: An Appraisal Based on Scientific Methodology. In D. Aston, B. Bader, C. Gallorini \& al. Under the Potter's Tree: Studies on Ancient Egypt Presented to Janine Bourriau. Leyde : Uitgeverij Peeters en Department Oosterse Studies Publishers (Orientalia Lovaniensia Analecta 204): 263-286.

Dunham, D. 1967 Second Cataract Forts, vol. II: Uronarti, Shalfak, Mirgissa. Boston : Museum of Fine Arts.

Épron, L., Daumas, Fr., Goyon, G. 1939 Le tombeau de Ti, fascicule I, Les approches de la chapelle, MIFAO 65/1. Gardiner, A.H. 1947 Ancient Egyptian Onomastica, 3 vol. Oxford : Oxford University Press.

Goyon, J.-Cl., Golvin, J.-Cl., Simon-Boidot, Cl. \& al. 2004 La Construction pharaonique. Paris : Picard.

Grenfell, B.P., Hunt, A.S. 1901 The Amherst Papyri Being an Account of the Greek Papyri in the Collection of the Right Hon. Lord Amherst of Hackney, F. S. A. at Didlington hall, Norfolk, II. London : Oxford University Press - Bernard Quaritch.

Grenier, J.-Cl. 1979 Djédem dans les textes du temple de Tôd. In J. Vercoutter Hommages à la mémoire de Serge Sauneron I. Le Caire: Institut français d'archéologie orientale (Bibliothèque d'étude 81) : 381-389.

Hassan, S. 1936 Excavations at Gîza, II, Le Caire : Government Press.

Hikade, T., Pyke, G., O'Neill, D. 2008 Excavations at Hierakonpolis HK29B and HK25: The campaigns of 2005/2006. Mitteilungen des Deutschen Archäologischen Instituts Abteilung Kairo 64: 153-188.

Lefébure, G. 1886 Les hypogées royaux de Thèbes. Première division. Le tombeau de Séti Ier, MMAF 2.

Leitz, Chr. \& al. 2002 Lexikon der ägyptischen Götter und Götterbezeichnungen VI. Leuven-Paris : Dudley (Orientalia Lovaniensia Analecta 115).

Naville, E. 1886 Das aegyptische Todtenbuch der XVIII. bis XX. Berlin : Dynastie, 3 vol.

Nicholson, P. T., Shaw, I. 2000 Ancient Egyptian Materials and Technology. Cambridge : Cambridge University Press.

Morgan, J. de 1909 Kom Ombos III, Vienne : A. Holzhausen.

O'Connor, D. 1985 The "Cenotaphs" of the Middle Kingdom at Abydos. In P. Posener-Kriéger (éd.) Mélanges Gamal Eddin Mokhtar II. Le Caire: Institut français d'archéologie orientale (Bibliothèque d'étude 97): 161-177, fig. 5 et 6.

Orrieux, Cl. 1983 Les Papyrus de Zénon, L’horizon d’un Grec en Égypte au III siècle avant J.-C. Paris : Éditions Macula (Deucalion).

Reeves, C. 1992 Egyptian Medicine. Princes Risborough : Shire Publications (Shire Egyptology Series 15).

Ritner, R. K. 1993 The Mechanics of Ancient Egyptian Magical Practice. Chicago : The Oriental Institute of the University of Chicago (Studies in Ancient Oriental Civilization 54). 
Ruffer, M. A. 1921 Studies in The Paleopathology of Ancient Egypt. Chicago : The University of Chicago Press. Sethe, K. 1908-1922 Die altägyptischen Pyramidentexte, nach den Papierabdrücken und Photographien des Berliner Museums, 4 vol. Leipzig : J. C. Hinrichs'sche Buchhandlung.

Yoyotte, J., Charvet, P., Gompertz, S. 1997 Strabon. Le Voyage en Égypte : un regard romain. Paris : Nil éditions (Le Cabinet de curiosités).

Vernière, Y. 1993 Diodore de Sicile. In F. Chamoux, P. Bertrac (dir.) Bibliothèque historique, Livre I (Collection des Universités de France, Budé). Paris : Les Belles Lettres.

Zandee, J. 1947 De Hymnen aan Amon van Papyrus Leiden I 350. Leyde : Oudheidkundige Mededelingen van het Rijksmuseum van Oudheden te Leiden 28.

Zignani, P. 2008 Enseignement d'un temple égyptien: conception architectonique du temple d'Hathor à Dendara. Lausanne : Presses Polytechniques et Universitaires Romandes.

Zivie, Chr. M. 1976 Giza au deuxième millénaire. Le Caire : Institut français d'archéologie orientale (Bibliothèque d'étude 70).

\section{POUR CITER CET ARTICLE}

Misuriello, J. 2014 La double perception du sable en Égypte ancienne, in S. Boulay \& M.-L. Gélard, Vivre le sable! Corps, matière et sociétés, Techniques \& Culture 61: 42-59.

\section{RÉSUMÉ}

La double perception du sable en Égypte ancienne. L'Égypte est une oasis dans une immense mer de sable: $96 \%$ de la surface du pays est désertique. Au gré des changements climatiques, la population s'est progressivement regroupée sur une mince bande de terre fertile formée par la vallée du Nil. Lorsqu'on s'intéresse au rapport des Égyptiens avec le sable, il faut toujours garder à l'esprit cette situation géographique particulière. Le sable constitue l'élément principal du milieu hostile et méconnu qui borde la terre fertile et familière de la Vallée. Se jouant des frontières, le sable, porté par le vent, pénètre également l'espace vital de l'Égyptien. Le lexique va ainsi expliciter la réalité d'un paysage contrasté en distinguant le sable de la rive et le sable du désert. L'étude proposée ici repose essentiellement sur l'analyse des textes, témoignages précieux laissés en abondance par une civilisation où l'écrit avait une place fondamentale. Il s'agit de réfléchir sur l'originalité de l'interaction entre les Égyptiens et le sable: comment a-t-on cohabité au quotidien avec cet élément du paysage, se l'est-on approprié, par quels moyens, et finalement comment l'a-t-on perçu? Plusieurs approches sont envisagées dans la première partie: conséquences de l'omniprésence du sable sur les espaces domestiqués et intégration aux activités du quotidien, influence sur le corps humain. Les réponses apportées à la problématique de cohabitation avec cet élément naturel ainsi que les procédés techniques mis en place à partir du sable considéré comme un matériau sont également examinés.

Le deuxième volet de l'étude concerne le sable dans les textes sacrés. Différents rituels faisant intervenir du sable ainsi que des épisodes mythiques citant l'élément sont discutés. Il apparaît clairement que l'ambiguité issue de l'expérience quotidienne, sable fertile mais aussi, par opposition, sable hostile voire dangereux, est transposée dans le discours religieux. Selon le contexte, des valeurs positives (protection, pureté, sacralité, fertilité), ou au contraire négatives (dangerosité, hostilité, léthargie, infertilité, stérilité, entrave au déplacement) lui seront attribuées. En dernier lieu, l'interrogation porte sur le statut accordé au sable. À la fois solide et liquide, matière mouvante et fluide, sa nature même implique-t-elle l'attribution d'un statut particulier par rapport aux autres éléments naturels, également intégrés au discours mythique? 


\section{ABSTRACT}

The double perception of sand in Ancient Egypt. Egypt looks like a haven amidst a huge sand sea - 96\% of the surface of the country is a desert. According to climate changes, the population has gradually gathered on a thin strip of fertile land: the Nile Valley. While looking at the connection between the Egyptians and the sand, one has to keep in mind this peculiar geographic situation. The sand represents the main component of the hostile and barren environment which surrounds the fertile and familiar land of the Valley. Making light of the borders, carried by the wind, the sand also enters the Egyptian's life space. Thus, the lexicon expresses the reality of a landscape with sharp contrasts by distinguishing the sand of the river from the sand of the desert. This study mainly relies on the analysis of texts, valuable testimonies left in profusion by a civilisation for which writing was fundamental. The aim is to think the originality of the interaction between the Egyptians and the sand: how did people manage to daily live with it? Did they appropriate it and how? Finally, how was it perceived? Several points are developed in the first part: consequences of the omnipresence of the sand for domesticated spaces and human activities, impact on human body. The answers brought to the problem of living with this natural element and the technical processes based on sand as a material are also under examination. The second part of the study deals with the sand in sacred texts. Several rituals including sand along with myths quoting the element are discussed. It clearly appears that the ambiguity coming from daily experience - fertile sand but also, on the contrary, hostile and even dangerous sand - can be found in the religious speech too. According to the text backgrounds, sand is linked either with positive values (protection, purity, sacrality, fertility), or negative ones (dangerousness, hostility, lethargy, infertility, sterility, hindrance to motion). At last, the question is the status given to the sand. Solid and liquid, a shifting and fluid material, does its very nature imply the attribution of a particular status compared to the other natural elements which are also present in the mythical speech?

\section{MOTS CLÉS}

Sable, Égypte ancienne, archéologie, textes, discours religieux, dualité

\section{KEYWORDS}

Sand, ancient Egypt, archaeology, texts, religious speech, duality 